\title{
A Closed-Form Full-State Feedback Controller for Stabilization of 3D Magnetohydrodynamic Channel Flow
}

\author{
Rafael Vazquez* \\ Departamento de Ingeniería Aeroespacial \\ Universidad de Sevilla \\ C. Descubrimientos, 41092 Sevilla, Spain \\ Eugenio Schuster ${ }^{\dagger}$ \\ Dept. Mechanical Eng. \& Mechanics \\ Lehigh University \\ Bethlehem, PA 18015-1835, USA \\ Miroslav Krstic \\ Department of Mechanical and Aerospace Engineering \\ University of California, San Diego \\ La Jolla, California 92093-0411, USA
}

\begin{abstract}
We present a boundary feedback law that stabilizes the velocity, pressure, and electromagnetic fields in a magnetohydrodynamic (MHD) channel flow. The MHD channel flow, also known as Hartmann flow, is a benchmark for applications such as cooling, hypersonic flight and propulsion. It involves an electrically conducting fluid moving between parallel plates in the presence of an externally imposed transverse magnetic field. The system is described by the inductionless MHD equations, a combination of
\end{abstract}

*Corresponding author: Address: Departamento de Ingeniería Aeroespacial, Universidad de Sevilla, C. Descubrimientos, 41092 Sevilla, Spain; phone: (34)-954-488-148; e-mail: rvazquez1@us .es

${ }^{\dagger}$ e-mail:schuster@lehigh.edu

${ }^{\ddagger}$ - - mail:krstic@ucsd.edu 
the Navier-Stokes equations and a Poisson equation for the electric potential under the MHD approximation in a low magnetic Reynolds number regime. This model is unstable for large Reynolds numbers, and is stabilized by actuation of velocity and the electric potential at only one of the walls. The backstepping method for stabilization of parabolic PDEs is applied to the velocity field system written in appropriate coordinates. Control gains are computed by solving a set of linear hyperbolic PDEs. Stabilization of non-discretized 3-D MHD channel flow has so far been an open problem.

\section{Nomenclature}

j Electric current.

$k_{x}, k_{y} \quad$ Wave numbers.

$K, \Gamma_{1}, \Gamma_{2}$ Control kernels.

$H \quad$ Hartmann number.

$N \quad$ Stuart number.

P Pressure.

p Pressure fluctuation.

Re Reynolds number.

$R e_{M} \quad$ Magnetic Reynolds number.

U Streamwise velocity.

$U^{e} \quad$ Streamwise equilibrium velocity.

$U_{C} \quad$ Streamwise velocity controller.

$u \quad$ Streamwise velocity fluctuation. 
V Wall-normal velocity.

$V_{C} \quad$ Wall-normal velocity controller.

W Spanwise velocity.

$W_{C} \quad$ Spanwise velocity controller.

$\phi \quad$ Electric potential.

$\Phi_{C} \quad$ Electric potential controller.

$\Lambda \quad$ Lyapunov function.

\section{Introduction}

In this paper, we derive an explicit boundary controller that stabilizes an incompressible magnetohydrodynamic (MHD) flow in an infinite rectangular 3-D channel. Known as the Hartmann flow [1], this model is considered a benchmark for applications such as liquidmetal cooling of nuclear reactors and supercomputers, plasma confinement, electromagnetic casting, hypersonic flight and propulsion.

In the Hartmann flow, an electrically conducting fluid (such as a liquid metal, a plasma or salt water) moves between parallel plates and is affected by an imposed transverse magnetic field. The movement of a conducting fluid produces an electric field and subsequently an electric current. The interaction between this current and the external magnetic field induces a body force, called the Lorentz force, which acts on the fluid itself. Hence the velocity and electromagnetic fields are highly coupled. These fields are mathematically described by the MHD equations [2], which are the Navier-Stokes equation coupled with the Maxwell equations. 
In the non-conducting case, the geometry we consider (channel flow) is known to be unstable for high Reynolds numbers and has been thoroughly studied and is frequently cited as a paradigm for transition to turbulence [3]. There are many works in flow control that consider the problem of channel flow stabilization, for instance, using optimal control [4], backstepping [5, 6], spectral decomposition/pole placement [7], Lyapunov design/passivity [8, 9], or nonlinear model reduction/in-domain actuation [10].

The stability of the Hartmann flow has also been extensively studied, both from the numerical and the analytical point of view $[11,12,13,14]$. However, specific results on stabilization of magnetohydrodynamic flows are more scarce. Prior works focuse mainly on electro-magneto-hydro-dynamic (EMHD) flow control in weak electrically conducting fluids such as saltwater. Traditionally two types of actuator designs have been used: one type generates a Lorentz field parallel to the wall in the streamwise direction [15], while the other one generates a Lorentz field normal to the wall in the spanwise direction $[16$, 17]. EMHD flow control has been dominated by strategies that either permanently activate the actuators or pulse them at arbitrary frequencies. However, it has been shown that feedback control schemes can improve the efficiency, by reducing control power, for both streamwise [18] and spanwise [19, 20] approaches. Other recent developments use modelbased techniques, for instance using nonlinear model reduction [21, 22] or optimal control [23]. There are some experimental results are available as well, showing the feasibility of MHD flow control; actuators consist of magnets and electrodes [16, 17, 24], for instance electromagnetic tiles [25]. Mathematical studies of controllability of magnetohydrodynamic flows have been done, though they do not provide explicit controllers [26, 27].

Applications include drag reduction [16, 20], boundary layer control [25, 28], mixing Rafael Vazquez DS-08-1170 
enhancement for cooling systems [29, 30], turbulence control [31], or estimation of velocity, pressure and electromagnetic fields [32].

This paper uses the backstepping method and extends our previous work for stabilization of the velocity field in a (non-conducting) 3-D channel flow [6]. It also extends to three dimensions our past efforts for the 2-D Hartmann flow [33]. None of these extensions are trivial, since the growing number of states (three components of the velocity field, the electric field and the pressure field, all infinite-dimensional, evolving in an infinite 3-D region) make the problem very challenging.

Our controller is designed for the continuum MHD model. Since the system is spatially invariant [34], control synthesis is done in the wave number space after application of a Fourier transform. Large wave numbers are found to be stable and left uncontrolled whereas for small wave numbers control is used. For these wave numbers, control is used to put the system in a strict-feedback form; this is necessary for application of the backstepping method for stabilization of parabolic PDE's [35]. Writing the velocity field in some appropriate coordinates, the resulting system is very similar to the Orr-Sommerfeld-Squire system of PDE's for non-conducting fluids and presents the same difficulties (non-normality leading to a large transient growth mechanism $[36,37])$. Thus, applying the same ideas as in [6], we use backstepping not only to guarantee stability but also to decouple the system in order to prevent transients. The control gains are computed solving a set of linear hyperbolic PDEs - a much simpler task than, for instance, solving nonlinear operator Riccati equations. Actuation of velocity and electric potential is done at only one of the channel walls. Full state knowledge is assumed, but the controller can be combined with an observer for MHD channel flow [32], which is a dual to the controller design in the present paper, to obtain an 


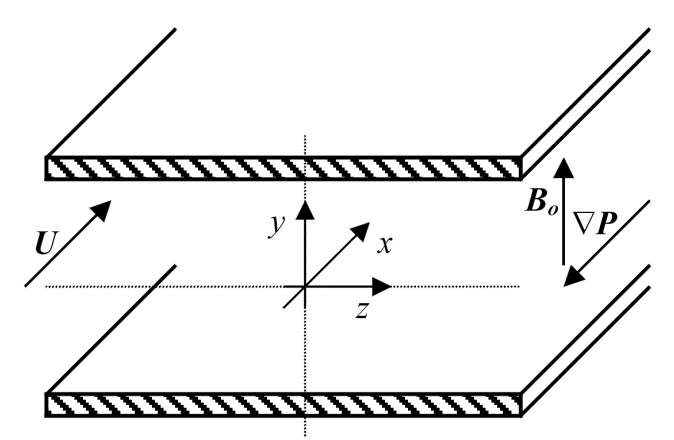

Figure 1: Hartmann Flow.

output feedback controller.

The paper is organized as follows. Section 2 introduces the governing equations. The equilibrium profile is presented in Section 3 and the linearized plant in wave number space introduced in Section 4. Section 5 presents the design of the control laws to guarantee stability of the closed-loop system and states the main result. We end the paper with concluding remarks in Section 6.

\section{Model}

Consider an incompressible conducting fluid enclosed between two plates, separated by a distance $L$, under the influence of a pressure gradient $\nabla P$ and a magnetic field $B_{0}$ normal to the walls, as shown in Figure 1. Under the assumption of a very small magnetic Reynolds number

$$
R e_{M}=\nu \rho \sigma U_{0} L \ll 1
$$

where $\nu$ is the viscosity of the fluid, $\rho$ the density of the fluid, $\sigma$ the conductivity of the fluid, and $U_{0}$ the reference velocity (maximum velocity of the equilibrium profile), the dynamics 
of the magnetic field can be neglected and the dimensionless velocity and electric potential field is governed by the inductionless MHD equations [38].

We set nondimensional coordinates $(x, y, z)$, where $x$ is the streamwise direction (parallel to the pressure gradient), $y$ the wall normal direction (parallel to the magnetic field) and $z$ the spanwise direction, so that $(x, y, z) \in(-\infty, \infty) \times[0,1] \times(-\infty, \infty)^{1}$. The governing equations are

$$
\begin{aligned}
U_{t} & =\frac{\triangle U}{R e}-U U_{x}-V U_{y}-W U_{z}-P_{x}+N \phi_{z}-N U \\
V_{t} & =\frac{\triangle V}{R e}-U V_{x}-V V_{y}-W V_{z}-P_{y} \\
W_{t} & =\frac{\triangle W}{R e}-U W_{x}-V W_{y}-W W_{z}-P_{z}-N \phi_{x}-N W \\
\triangle \phi & =U_{z}-W_{x}
\end{aligned}
$$

where $U, V$ and $W$ denote, respectively, the streamwise, wall-normal and spanwise velocities, $P$ the pressure, $\phi$ the electric potential, $R e=\frac{U_{0} L}{\nu}$ is the Reynolds number and $N=\frac{\sigma L B_{0}^{2}}{\rho U_{0}}$ the Stuart number. Since the fluid is incompressible, the continuity equation is verified

$$
U_{x}+V_{y}+W_{z}=0
$$

The boundary conditions for the velocity field are

$$
\begin{aligned}
U(t, x, 0, z) & =0, U(t, x, 1, z)=U_{c}(t, x, z), \\
V(t, x, 0, z) & =0, V(t, x, 1, z)=V_{c}(t, x, z), \\
W(t, x, 0, z) & =0, W(t, x, 1, z)=W_{c}(t, x, z),
\end{aligned}
$$

\footnotetext{
${ }^{1}$ Our approach can be extended to finite, periodic channels with only some changes; see e.g. [39] for techniques involved.
} 
where $U_{c}(t, x, z), V_{c}(t, x, z)$ and $W_{c}(t, x, z)$ denote, respectively, the actuators for streamwise, wall-normal and spanwise velocity in the upper wall. We denote the initial conditions for the velocity field as $U_{0}(x, y, z)=U(0, x, y, z), V_{0}(x, y, z)=V(0, x, y, z), W_{0}(x, y, z)=$ $W(0, x, y, z)$.

Assuming perfectly conducting walls, the electric potential must verify

$$
\phi(t, x, 0, z)=0, \phi(t, x, 1, z)=\Phi_{c}(t, x, z),
$$

where $\Phi_{c}(t, x, z)$ is the imposed potential (electromagnetic actuation) in the upper wall. The nondimensional electric current, $j(t, x, y, z)$, a vector field that is computed from the electric potential and velocity fields as follows,

$$
\begin{aligned}
& j^{x}(t, x, y, z)=-\phi_{x}-W, \\
& j^{y}(t, x, y, z)=-\phi_{y}, \\
& j^{z}(t, x, y, z)=-\phi_{z}+U,
\end{aligned}
$$

where $j^{x}, j^{y}$, and $j^{z}$ denote the components of $j$.

We assume that all actuators can be independently actuated for every $(x, z) \in \mathbb{R}^{2}$. Note that no actuation is done inside the channel or at the bottom wall.

\section{Equilibrium profile}

The equilibrium profile for system (2)-(5) with no control can be calculated following the same steps that yield the Poiseuille solution for Navier-Stokes channel flow. Thus, we assume a steady solution with only one nonzero nondimensional velocity component, $U^{e}(y)$, that 
depends only on the $y$ coordinate. Substituting $U^{e}(y)$ in equation (2), one finds that it verifies the following equation,

$$
0=\frac{U_{y y}^{e}(y)}{R e}-P_{x}^{e}-N U^{e}(y)
$$

whose nondimensional solution is, setting $P^{e}$ such that the maximum velocity (centerline velocity) is unity,

$$
\begin{aligned}
U^{e}(y) & =\frac{\sinh (H(1-y))-\sinh H+\sinh (H y)}{2 \sinh H / 2-\sinh H}, \\
V^{e} & =W^{e}=\phi^{e}=0 \\
P^{e} & =\frac{N \sinh H}{2 \sinh H / 2-\sinh H} x, \\
j^{x e} & =j^{y e}=0, j^{z e}=U^{e}(y) .
\end{aligned}
$$

where $H=\sqrt{\operatorname{ReN}}=B_{0} L \sqrt{\frac{\sigma}{\rho \nu}}$ is the Hartmann number. In Fig. 2(left) we show $U^{e}(y)$ for different values of $H$. Since the equilibrium profile is nondimensional the centerline velocity is always 1. For $H=0$ the classic parabolic Poiseuille profile is recovered. In Fig. 2 (right) we show $U_{y}^{e}(y)$, proportional to shear stress, whose maximum is reached at the boundaries and grows with $H$.

\section{The Plant in Wave Number Space}

Define the fluctuation variables

$$
\begin{aligned}
& u(t, x, y, z)=U(t, x, y, z)-U^{e}(y), \\
& p(t, x, y, z)=P(t, x, y, z)-P^{e}(y)
\end{aligned}
$$

where $U^{e}(y)$ and $P^{e}(y)$ are, respectively, the equilibrium velocity and pressure given in (15) and (17). The linearization of (2)-(4) around the Hartmann equilibrium profile, written in 

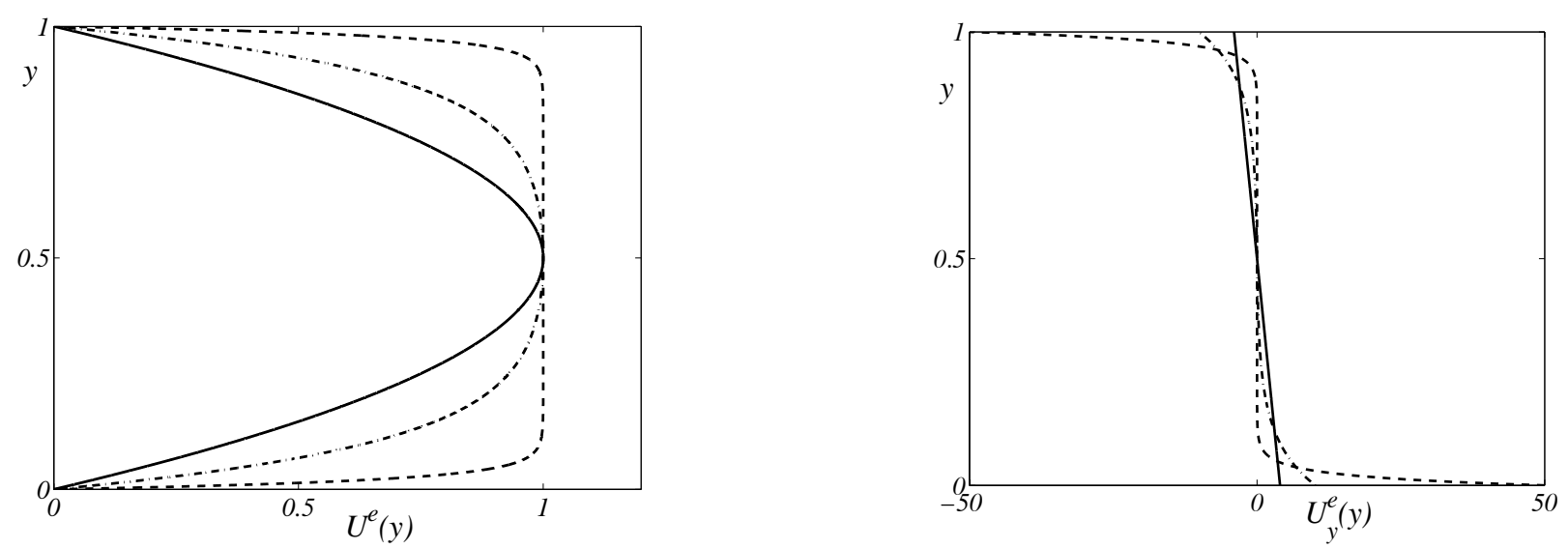

Figure 2: Streamwise equilibrium velocity $U^{e}(y)$ (left) and $U_{y}^{e}(y)$ (right), for different values of $H$. Solid, $H=0$; dash-dotted, $H=10$; dashed, $H=50$.

the fluctuation variables $(u, V, W, p, \phi)$, is

$$
\begin{aligned}
u_{t} & =\frac{\triangle u}{R e}-U^{e}(y) u_{x}-U_{y}^{e}(y) V-p_{x}+N \phi_{z}-N u, \\
V_{t} & =\frac{\triangle V}{R e}-U^{e}(y) V_{x}-p_{y}, \\
W_{t} & =\frac{\triangle W}{R e}-U^{e}(y) W_{x}-p_{z}-N \phi_{x}-N W .
\end{aligned}
$$

The equation for the potential is

$$
\triangle \phi=u_{z}-W_{x}
$$

and the fluctuation velocity field verifies the continuity equation,

$$
u_{x}+V_{y}+W_{z}=0
$$


and the following boundary conditions

$$
\begin{aligned}
u(t, x, 0, z) & =W(t, x, 0, z)=V(t, x, 0, z)=0, \\
u(t, x, 1, z) & =U_{c}(t, x, z) \\
V(t, x, 1, z) & =V_{c}(t, x, z) \\
W(t, x, 1, z) & =W_{c}(t, x, z), \\
\phi(t, x, 0, z) & =0, \phi(t, x, 1, z)=\Phi_{c}(t, x, z) .
\end{aligned}
$$

We denote the initial conditions for the fluctuation velocity as $u_{0}(x, y, z)=U_{0}(x, y, z)-$ $U^{e}(y)$

To guarantee stability, our design task is to design feedback laws $U_{c}, V_{c}, W_{c}$ and $\Phi_{c}$, so that the origin of the velocity fluctuation system is exponentially stable. Full state knowledge is assumed.

Since the plant is linear and spatially invariant [34], we use a Fourier transform in the $x$ and $z$ coordinates (the spatially invariant directions). The transform pair (direct and inverse transform) is defined for any function $f(x, y, z)$ as

$$
\begin{aligned}
f\left(k_{x}, y, k_{z}\right) & =\int_{-\infty}^{\infty} \int_{-\infty}^{\infty} f(x, y, z) \mathrm{e}^{-2 \pi i\left(k_{x} x+k_{z} z\right)} d z d x \\
f(x, y, z) & =\int_{-\infty}^{\infty} \int_{-\infty}^{\infty} f\left(k_{x}, y, k_{z}\right) \mathrm{e}^{2 \pi i\left(k_{x} x+k_{z} z\right)} d k_{z} d k_{x} .
\end{aligned}
$$

Note that we use the same symbol $f$ for both the original $f(x, y, z)$ and the image $f\left(k_{x}, y, k_{z}\right)$. In hydrodynamics $k_{x}$ and $k_{z}$ are referred to as the "wave numbers." 
The plant equations in wave number space are

$$
\begin{aligned}
u_{t}= & \frac{-\alpha^{2} u+u_{y y}}{R e}-\beta(y) u-U_{y}^{e}(y) V-2 \pi k_{x} i p-N u \\
& +2 \pi k_{z} i N \phi, \\
V_{t}= & \frac{-\alpha^{2} V+V_{y y}}{R e}-\beta(y) V-p_{y}, \\
W_{t}= & \frac{-\alpha^{2} W+W_{y y}}{R e}-\beta(y) W-2 \pi k_{z} i p-N W \\
& -2 \pi k_{x} i N \phi
\end{aligned}
$$

where $\alpha^{2}=4 \pi^{2}\left(k_{x}^{2}+k_{z}^{2}\right)$ and $\beta(y)=2 \pi i k_{x} U^{e}(y)$.

The continuity equation in wave number space is expressed as

$$
2 \pi i k_{x} u+V_{y}+2 \pi k_{z} W=0
$$

and the equation for the potential is

$$
-\alpha^{2} \phi+\phi_{y y}=2 \pi i\left(k_{z} u-k_{x} W\right) .
$$

The boundary conditions are

$$
\begin{aligned}
u\left(t, k_{x}, 0, k_{z}\right) & =W\left(t, k_{x}, 0, k_{z}\right)=V\left(t, k_{x}, 0, k_{z}\right)=0, \\
u\left(t, k_{x}, 1, k_{z}\right) & =U_{c}\left(t, k_{x}, k_{z}\right), \\
V\left(t, k_{x}, 1, k_{z}\right) & =V_{c}\left(t, k_{x}, k_{z}\right), \\
W\left(t, k_{x}, 1, k_{z}\right) & =W_{c}\left(t, k_{x}, k_{z}\right), \\
\phi\left(t, k_{x}, 0, k_{z}\right) & =0, \phi\left(t, k_{x}, 1, k_{z}\right)=\Phi_{c}\left(t, k_{x}, k_{z}\right) .
\end{aligned}
$$




\section{Control Design}

We design the controller in wave number space. Note that (33)-(42) are uncoupled for each wave number. It is well known that large wave numbers (which correspond to small scales where dissipation is present) are already stable and instability can only be found in small wave numbers (large scale behavior) [3]. Therefore, as in [5, 6], the set of wave numbers $k_{x}^{2}+k_{z}^{2} \leq M^{2}$, which we refer to as the controlled wave number range, and the set $k_{x}^{2}+k_{z}^{2}>M^{2}$, the uncontrolled wave number range, can be treated and studied separately. If stability for all wave numbers is established, stability in physical space follows (see [5]). The number $M$, which will be computed in Section 5.2, is a parameter that ensures stability for uncontrolled wave numbers.

We define $\chi$, a truncating function, as

$$
\chi\left(k_{x}, k_{z}\right)=\left\{\begin{array}{cc}
1, & k_{x}^{2}+k_{z}^{2} \leq M^{2}, \\
0, & \text { otherwise. }
\end{array}\right.
$$

Then, we reflect that we don't use control for large wave numbers by setting

$$
\left(\begin{array}{c}
U_{c}(t, x, z) \\
V_{c}(t, x, z) \\
W_{c}(t, x, z) \\
\Phi_{c}(t, x, z)
\end{array}\right)=\int_{-\infty}^{\infty} \int_{-\infty}^{\infty}\left(\begin{array}{c}
U_{c}\left(t, k_{x}, k_{z}\right) \\
V_{c}\left(t, k_{x}, k_{z}\right) \\
W_{c}\left(t, k_{x}, k_{z}\right) \\
\Phi_{c}\left(t, k_{x}, k_{z}\right)
\end{array}\right)
$$

Next we design stabilizing control laws for small wave numbers and analyze uncontrolled wave numbers. 


\subsection{Controlled wave number analysis}

Consider $k_{x}^{2}+k_{z}^{2} \leq M^{2}$. Then $\chi=1$, so there is control. Using the continuity equation (36) and taking divergence of (33)-(35), a Poisson equation for the pressure is derived,

$$
-\alpha^{2} p+p_{y y}=-4 \pi k_{x} i U_{y}^{e}(y) V+N V_{y}
$$

Evaluating equation (34) at $y=0$ one finds that

$$
p_{y}\left(k_{x}, 0, k_{z}\right)=\frac{V_{y y}\left(k_{x}, 0, k_{z}\right)}{R e}=-2 \pi i \frac{k_{x} u_{y 0}+k_{z} W_{y 0}}{R e},
$$

where we use (36) for expressing $V_{y y}$ at the bottom in terms of $u_{y 0}=u_{y}\left(k_{x}, 0, k_{z}\right)$ and $W_{y 0}=W_{y}\left(k_{x}, 0, k_{z}\right)$. Similarly, evaluating equation (34) at $y=1$ we get

$$
\begin{aligned}
p_{y}\left(k_{x}, 1, k_{z}\right) & =\frac{V_{y y}\left(k_{x}, 1, k_{z}\right)}{R e}-\left(V_{c}\right)_{t}-\alpha^{2} \frac{V_{c}}{R e} \\
& =-2 \pi i \frac{k_{x} u_{y 1}+k_{z} W_{y 1}}{R e}-\left(V_{c}\right)_{t}-\alpha^{2} \frac{V_{c}}{R e}
\end{aligned}
$$

where we use (36) for expressing $V_{y y}$ at the top wall in terms of $u_{y 1}=u_{y}\left(k_{x}, 1, k_{z}\right)$ and $W_{y 1}=W_{y}\left(k_{x}, 1, k_{z}\right)$ and the controller $V_{c}$.

Equation (45) can be solved in terms of integrals of the state and the boundary terms 
appearing in (46) and (47).

$$
\begin{aligned}
p= & -\frac{4 \pi k_{x} i}{\alpha} \int_{0}^{y} U_{y}^{e}(\eta) \sinh (\alpha(y-\eta)) V\left(k_{x}, \eta, k_{z}\right) d \eta \\
& +N \int_{0}^{y} \frac{\sinh (\alpha(y-\eta))}{\alpha} V_{y}\left(k_{x}, \eta, k_{z}\right) d \eta \\
& +2 \pi i \frac{\cosh (\alpha(1-y))}{\alpha \sinh \alpha} \frac{k_{x} u_{y 0}+k_{z} W_{y 0}}{R e} \\
& +\frac{4 \pi k_{x} i \cosh (\alpha y)}{\alpha \sinh \alpha} \int_{0}^{1} U_{y}^{e}(\eta) \cosh (\alpha(1-\eta)) \\
& \times V\left(k_{x}, \eta, k_{z}\right) d \eta-N \frac{\cosh (\alpha y)}{\alpha \sinh \alpha} \int_{0}^{1} \cosh (\alpha(1-\eta)) \\
& \times V_{y}\left(k_{x}, \eta, k_{z}\right) d \eta-2 \pi i \frac{\cosh (\alpha y)}{\alpha \sinh \alpha} \frac{k_{x} u_{y 1}+k_{z} W_{y 1}}{R e} \\
& -\frac{\cosh (\alpha y)}{\alpha \sinh \alpha}\left(\left(V_{c}\right)_{t}+\alpha^{2} \frac{V_{c}}{R e}\right) .
\end{aligned}
$$

We proceed as in [5] and [6] and use the controller $V_{c}$, which appears inside the pressure solution (48), to make the pressure strict-feedback (spatially causal in $y$ ), which is a necessary structure for the application of a backstepping boundary controller [35]. Physically this means that we do not let pressure perturbations of the flow at one given point to be affected by the velocity field closer than the point to the upper wall; only the velocity field "below" the point (closer to the lower wall) can have any affect on the pressure perturbation.

Since the first three lines in (48) are already spatially causal, we need to cancel the fourth, fifth and sixth lines of (48). Set

$$
\begin{aligned}
\left(V_{c}\right)_{t}= & \alpha^{2} \frac{V_{c}}{R e}+2 \pi i \frac{k_{x}\left(u_{y 0}-u_{y 1}\right)+k_{z}\left(W_{y 0}-W_{y 1}\right)}{R e} \\
& +4 \pi k_{x} i \int_{0}^{1} U_{y}^{e}(\eta) \cosh (\alpha(1-\eta)) V\left(k_{x}, \eta, k_{z}\right) d \eta \\
& -N \int_{0}^{1} \cosh (\alpha(1-\eta)) V_{y}\left(k_{x}, \eta, k_{z}\right) d \eta
\end{aligned}
$$


which can be written as

$$
\begin{aligned}
\left(V_{c}\right)_{t}= & \alpha^{2} \frac{V_{c}}{R e}+2 \pi i \frac{k_{x}\left(u_{y 0}-u_{y 1}\right)+k_{z}\left(W_{y 0}-W_{y 1}\right)}{R e} \\
& -N V_{c}+\int_{0}^{1} \cosh (\alpha(1-\eta)) V\left(k_{x}, \eta, k_{z}\right) \\
& \times\left(N+4 \pi k_{x} i U_{y}^{e}(\eta)\right) d \eta .
\end{aligned}
$$

Then, the pressure is written in terms of a strict-feedback integral of the state $V$ and the boundary terms $u_{y 0}, W_{y 0}$ (proportional to the skin friction at the bottom) as follows

$$
\begin{aligned}
p= & -\frac{4 \pi k_{x} i}{\alpha} \int_{0}^{y} U_{y}^{e}(\eta) \sinh (\alpha(y-\eta)) V\left(k_{x}, \eta, k_{z}\right) d \eta \\
& -2 \pi i \frac{\cosh (\alpha y)-\cosh (\alpha(1-y))}{\operatorname{Re} \alpha \sinh \alpha}\left(k_{x} u_{y 0}+k_{z} W_{y 0}\right) \\
& +N \int_{0}^{y} \frac{\sinh (\alpha(y-\eta))}{\alpha} V_{y}\left(k_{x}, \eta, k_{z}\right) d \eta
\end{aligned}
$$

Similarly, solving for $\phi$ in terms of the control $\Phi_{c}$ and the right hand side of its Poisson equation (37),

$$
\begin{aligned}
\phi= & \frac{2 \pi i}{\alpha} \int_{0}^{y} \sinh (\alpha(y-\eta))\left(k_{z} u\left(k_{x}, \eta, k_{z}\right)\right. \\
& \left.-k_{x} W\left(k_{x}, \eta, k_{z}\right)\right) d \eta+\frac{\sinh (\alpha y)}{\sinh \alpha} \Phi_{c}\left(k_{x}, k_{y}\right) \\
& -\frac{2 \pi i \sinh (\alpha y)}{\alpha \sinh \alpha} \int_{0}^{1} \sinh (\alpha(1-\eta))\left(k_{z} u\left(k_{x}, \eta, k_{z}\right)\right. \\
& \left.-k_{x} W\left(k_{x}, \eta, k_{z}\right)\right) d \eta
\end{aligned}
$$

As in the pressure, an actuator $\left(\Phi_{c}\right.$ in this case) appears inside the solution for the potential. The last two lines of (52) are non-strict-feedback integrals and need to be cancelled to apply the backstepping method. As for the pressure, this means that we do not let electric field perturbations of the flow at one given point to be affected by the velocity field further "up" than the point (closer to the upper wall); only the velocity field closer to the lower wall affects the electrical field perturbation. 
For this we use $\Phi_{c}$ by setting

$$
\begin{aligned}
\Phi_{c}\left(k_{x}, k_{y}\right)= & \frac{2 \pi i}{\alpha} \int_{0}^{1} \sinh (\alpha(1-\eta))\left(k_{z} u\left(k_{x}, \eta, k_{z}\right)\right. \\
& \left.-k_{x} W\left(k_{x}, \eta, k_{z}\right)\right) d \eta
\end{aligned}
$$

Then the potential can then be expressed as a strict-feedback integral of the states $u$ and $W$ as follows

$$
\begin{aligned}
\phi= & \frac{2 \pi i}{\alpha} \int_{0}^{y} \sinh (\alpha(y-\eta))\left(k_{z} u\left(k_{x}, \eta, k_{z}\right)\right. \\
& \left.-k_{x} W\left(k_{x}, \eta, k_{z}\right)\right) d \eta .
\end{aligned}
$$

Introducing the expressions (51) and (54) in (33) and (35), we get

$$
\begin{aligned}
u_{t}= & \frac{-\alpha^{2} u+u_{y y}}{R e}-\beta(y) u-U_{y}^{e}(y) V-N u-4 \pi^{2} k_{x} \\
& \times \frac{\cosh (\alpha y)-\cosh (\alpha(1-y))}{\operatorname{Re} \alpha \sinh \alpha}\left(k_{x} u_{y 0}+k_{z} W_{y 0}\right) \\
& -\frac{8 \pi k_{x}^{2}}{\alpha} \int_{0}^{y} U_{y}^{e}(\eta) \sinh (\alpha(y-\eta)) V\left(k_{x}, \eta, k_{z}\right) d \eta \\
& -2 \pi i k_{x} N \int_{0}^{y} \frac{\sinh (\alpha(y-\eta))}{\alpha} V_{y}\left(k_{x}, \eta, k_{z}\right) d \eta \\
& -\frac{4 \pi^{2} k_{z} N}{\alpha} \int_{0}^{y} \sinh (\alpha(y-\eta)) \\
& \times\left(k_{z} U\left(k_{x}, \eta, k_{z}\right)-k_{x} W\left(k_{x}, \eta, k_{z}\right)\right) d \eta, \\
W_{t}= & -\alpha^{2} W+W_{y y}-\beta(y) W-N W-4 \pi^{2} k_{z} \\
& \times \frac{\cosh (\alpha y)-\cosh (\alpha(1-y))}{\operatorname{Re} \alpha \sinh \alpha}\left(k_{x} u_{y 0}+k_{z} W_{y 0}\right) \\
& -\frac{8 \pi k_{x} k_{z}}{\alpha} \int_{0}^{y} U_{y}^{e}(\eta) \sinh (\alpha(y-\eta)) V\left(k_{x}, \eta, k_{z}\right) d \eta \\
& -2 \pi i k_{z} N \int_{0}^{y} \frac{\sinh (\alpha(y-\eta))}{\alpha} V_{y}\left(k_{x}, \eta, k_{z}\right) d \eta \\
& +\frac{4 \pi^{2} k_{x} N}{\alpha} \int_{0}^{y} \sinh (\alpha(y-\eta)) \\
& \times\left(k_{z} U\left(k_{x}, \eta, k_{z}\right)-k_{x} W\left(k_{x}, \eta, k_{z}\right)\right) d \eta .
\end{aligned}
$$

Rafael Vazquez

DS-08-1170 
We have omitted the equation for $V$ since, from (36) and using the fact that $V\left(k_{x}, 0, k_{z}\right)=0$, $V$ is computed as

$$
V=-2 \pi i \int_{0}^{y}\left(k_{x} U\left(k_{x}, \eta, k_{z}\right)+k_{z} W\left(k_{x}, \eta, k_{z}\right)\right) d \eta
$$

Now we use the following change of variables and its inverse,

$$
\begin{aligned}
Y & =2 \pi i\left(k_{x} u+k_{z} W\right), \omega=2 \pi i\left(k_{z} u-k_{x} W\right), \\
u & =\frac{2 \pi i}{\alpha^{2}}\left(k_{x} Y+k_{z} \omega\right), W=\frac{2 \pi i}{\alpha^{2}}\left(k_{z} Y-k_{x} \omega\right) .
\end{aligned}
$$

Note that $\omega$ is the normal vorticity, whereas $Y=-V_{y}$, the rate of change of the velocity $V$ along the channel.

Defining $\epsilon=\frac{1}{R e}$ and the following functions

$$
\begin{aligned}
g_{1}= & 4 \pi i k_{x}\left\{\frac{U_{y}^{e}(y)}{2}+\int_{\eta}^{y} U_{y}^{e}(\sigma) \frac{\sinh (\alpha(y-\sigma))}{\alpha} d \sigma\right\} \\
& +N \alpha \sinh (\alpha(y-\sigma)), \\
g_{2}= & -\alpha \frac{\cosh (\alpha y)-\cosh (\alpha(1-y))}{\operatorname{Re} \sinh \alpha}, \\
h_{1}= & 2 \pi i k_{z} U_{y}^{e}, \\
h_{2}= & -N \alpha \sinh (\alpha(y-\eta)),
\end{aligned}
$$

equations (55)-(56) expressed in terms of $Y$ and $\omega$ are

$$
\begin{aligned}
Y_{t}= & \epsilon\left(-\alpha^{2} Y+Y_{y y}\right)-\beta(y) Y-N Y+g Y_{y 0} \\
& +\int_{0}^{y} f\left(k_{x}, y, \eta, k_{z}\right) Y\left(k_{x}, \eta, k_{z}\right) d \eta \\
\omega_{t}= & \epsilon\left(-\alpha^{2} \omega+\omega_{y y}\right)-\beta(y) \omega-N \omega \\
& +h_{1}(y) \int_{0}^{y} Y\left(k_{x}, \eta, k_{z}\right) d \eta \\
& +\int_{0}^{y} h_{2}(y, \eta) \omega\left(k_{x}, \eta, k_{z}\right) d \eta
\end{aligned}
$$

Rafael Vazquez 
where we have used the inverse change of variables (59) to express $u_{y 0}$ and $W_{y 0}$ in terms of $Y_{y 0}=Y_{y}\left(k_{x}, 0, k_{z}\right)$ as follows

$$
Y_{y 0}=2 \pi i\left(k_{x} u_{y 0}+k_{z} W_{y 0}\right)
$$

with boundary conditions

$$
\begin{aligned}
Y\left(t, k_{x}, 0, k_{z}\right) & =\omega\left(t, k_{x}, 0, k_{z}\right)=0 \\
Y\left(t, k_{x}, 1, k_{z}\right) & =Y_{c}\left(t, k_{x}, k_{z}\right) \\
\omega\left(t, k_{x}, 1, k_{z}\right) & =\omega_{c}\left(t, k_{x}, k_{z}\right)
\end{aligned}
$$

where

$$
\begin{aligned}
& Y_{c}=2 \pi i\left(k_{x} U_{c}+k_{z} W_{c}\right), \\
& \omega_{c}=2 \pi i\left(k_{z} U_{c}-k_{x} W_{c}\right) .
\end{aligned}
$$

Equations (64)-(65) are a coupled, strict-feedback plant, with integral and reaction terms. As in [6], a variant of the design presented in [35] can be used to stabilize the system using a double backstepping transformation. The transformation maps, for each $k_{x}$ and $k_{z}$, the variables $(Y, \omega)$ into the variables $(\Psi, \Omega)$, that verify the following family of heat equations (parameterized in $k_{x}, k_{z}$ )

$$
\begin{aligned}
& \Psi_{t}=\epsilon\left(-\alpha^{2} \Psi+\Psi_{y y}\right)-\beta(y) \Psi-N \psi, \\
& \Omega_{t}=\epsilon\left(-\alpha^{2} \Omega+\Omega_{y y}\right)-\beta(y) \Omega-N \Omega,
\end{aligned}
$$

with boundary conditions

$$
\begin{aligned}
& \Psi\left(k_{x}, 0, k_{z}\right)=\Psi\left(k_{x}, 1, k_{z}\right)=0 \\
& \Omega\left(k_{x}, 0, k_{z}\right)=\Omega\left(k_{x}, 1, k_{z}\right)=0 .
\end{aligned}
$$


The transformation is defined as follows,

$$
\begin{aligned}
\Psi= & Y-\int_{0}^{y} K\left(k_{x}, y, \eta, k_{z}\right) Y\left(k_{x}, \eta, k_{z}\right) d \eta \\
\Omega= & \omega-\int_{0}^{y} \Gamma_{1}\left(k_{x}, y, \eta, k_{z}\right) Y\left(k_{x}, \eta, k_{z}\right) d \eta \\
& -\int_{0}^{y} \Gamma_{2}\left(k_{x}, y, \eta, k_{z}\right) \omega\left(k_{x}, \eta, k_{z}\right) d \eta .
\end{aligned}
$$

Following $[35,5,6]$, the functions $K\left(k_{x}, y, \eta, k_{z}\right), \Gamma_{1}\left(k_{x}, y, \eta, k_{z}\right)$, and $\Gamma_{2}\left(k_{x}, y, \eta, k_{z}\right)$ are found as the solution of the following partial integro-differential equations,

$$
\begin{aligned}
\epsilon K_{y y}= & \epsilon K_{\eta \eta}+(\beta(y)-\beta(\eta)) K-f \\
& +\int_{\eta}^{y} f(\eta, \xi) K(y, \xi) d \xi \\
\epsilon \Gamma_{1 y y}= & \epsilon \Gamma_{1 \eta \eta}+(\beta(y)-\beta(\eta)) \Gamma_{1}-h_{1}+\int_{\eta}^{y} \Gamma_{2}(y, \xi) \\
& \times h_{1}(\xi) d \xi+\int_{\eta}^{y} f(\eta, \xi) \Gamma_{1}(y, \xi) d \xi \\
\epsilon \Gamma_{2 y y}= & \epsilon \Gamma_{2 \eta \eta}+(\beta(y)-\beta(\eta)) \Gamma_{2}-h_{2} \\
& +\int_{\eta}^{y} h_{2}(\xi, \eta) \Gamma_{2}(y, \xi) d \xi .
\end{aligned}
$$

Equations (78)-(80) are hyperbolic partial integro-differential equation in the region $\mathcal{T}=$ $\{(y, \eta): 0 \leq y \leq 1,0 \leq \eta \leq y\}$. Their boundary conditions are

$$
\begin{aligned}
K(y, y) & =-\frac{g(0)}{\epsilon} \\
K(y, 0) & =\frac{\int_{0}^{y} K(y, \eta) g(\eta) d \eta-g(y)}{\epsilon} \\
\Gamma_{1}(y, 0) & =\frac{\int_{0}^{y} \Gamma_{1}(y, \eta) g(\eta) d \eta}{\epsilon} \\
\Gamma_{1}(y, y) & =0, \Gamma_{2}(y, y)=0, \Gamma_{2}(y, 0)=0 .
\end{aligned}
$$

Remark 5.1. Equations (78)-(84) are well-posed and can be solved symbolically, by means of a successive approximation series, or numerically $[35,6]$. Note that $(78)$ and $(80)$ are 
autonomous. Hence, one must solve first for $K\left(k_{x}, y, \eta, k_{z}\right)$ and $\Gamma_{2}\left(k_{x}, y, \eta, k_{z}\right)$. Then the solution for $\Gamma_{2}$ is plugged in Equation 79 which then can be solved for $\Gamma_{1}\left(k_{x}, y, \eta, k_{z}\right)$.

Control laws $Y_{c}$ and $W_{c}$ are found evaluating (76)-(77) at $y=1$ and using (68)-(69) and (74)-(75), which yields

$$
\begin{aligned}
Y_{c}\left(t, k_{x}, k_{z}\right)= & \int_{0}^{1} K\left(k_{x}, 1, \eta, k_{z}\right) Y\left(k_{x}, \eta, k_{z}\right) d \eta \\
\omega_{c}\left(t, k_{x}, k_{z}\right)= & \int_{0}^{1} \Gamma_{1}\left(k_{x}, 1, \eta, k_{z}\right) Y\left(k_{x}, \eta, k_{z}\right) d \eta \\
& +\int_{0}^{1} \Gamma_{2}\left(k_{x}, 1, \eta, k_{z}\right) \omega\left(k_{x}, \eta, k_{z}\right) d \eta .
\end{aligned}
$$

Using (58)-(59) to write $(85)-(86)$ in $(u, W)$, we get

$$
\begin{aligned}
U_{c}= & \int_{0}^{1} K^{U u}\left(k_{x}, 1, \eta, k_{z}\right) u\left(k_{x}, \eta, k_{z}\right) d \eta \\
& +\int_{0}^{1} K^{U W}\left(k_{x}, 1, \eta, k_{z}\right) W\left(k_{x}, \eta, k_{z}\right) d \eta \\
W_{c}= & \int_{0}^{1} K^{W u}\left(k_{x}, 1, \eta, k_{z}\right) u\left(k_{x}, \eta, k_{z}\right) d \eta \\
& +\int_{0}^{1} K^{W W}\left(k_{x}, 1, \eta, k_{z}\right) W\left(k_{x}, \eta, k_{z}\right) d \eta,
\end{aligned}
$$

where

$$
\left(\begin{array}{c}
K^{U u} \\
K^{U W} \\
K^{W u} \\
K^{W W}
\end{array}\right)=\mathbf{A}\left(\begin{array}{c}
K\left(k_{x}, y, \eta, k_{z}\right) \\
\Gamma_{1}\left(k_{x}, y, \eta, k_{z}\right) \\
0 \\
\Gamma_{2}\left(k_{x}, y, \eta, k_{z}\right)
\end{array}\right)
$$


and where the matrix $\mathbf{A}$ is defined as

$$
\mathbf{A}=-\frac{4 \pi^{2}}{\alpha^{2}}\left(\begin{array}{cccc}
k_{x}^{2} & k_{x} k_{z} & k_{x} k_{z} & k_{z}^{2} \\
k_{x} k_{z} & k_{z}^{2} & -k_{x}^{2} & -k_{x} k_{z} \\
k_{x} k_{z} & -k_{x}^{2} & k_{z}^{2} & -k_{x} k_{z} \\
k_{z}^{2} & -k_{x} k_{z} & -k_{x} k_{z} & k_{x}^{2}
\end{array}\right) .
$$

Stability in the controlled wave number range follows from stability of (72)-(73) and the invertibility of the transformation (76)-(77). We get the following result, whose proof we sketch (see [6] for more details).

Proposition 5.1. For $k_{x}^{2}+k_{z}^{2} \leq M^{2}$, the equilibrium $u \equiv V \equiv W \equiv 0$ of system (33)-(42) with control laws (50), (53), (87)-(88) is exponentially stable in the $L^{2}$ norm, i.e.,

$$
\begin{aligned}
& \int_{0}^{1}\left(|u|^{2}+|V|^{2}+|W|^{2}\right)\left(t, k_{x}, y, k_{z}\right) d y \\
\leq & C_{1} \mathrm{e}^{-2 \epsilon t} \int_{0}^{1}\left(\left|u_{0}\right|^{2}+\left|V_{0}\right|^{2}+\left|W_{0}\right|^{2}\right)\left(0, k_{x}, y, k_{z}\right) d y
\end{aligned}
$$

where $C_{1} \geq 0$.

Proof. From equations (72)-(73) we get, using a standard Lyapunov argument,

$$
\begin{aligned}
& \int_{0}^{1}\left(|\Psi|^{2}+|\Omega|^{2}\right)\left(t, k_{x}, y, k_{z}\right) d y \\
\leq & \mathrm{e}^{-2 \epsilon t} \int_{0}^{1}\left(|\Psi|^{2}+|\Omega|^{2}\right)\left(0, k_{x}, y, k_{z}\right) d y
\end{aligned}
$$

and then from the transformation (76)-(77) and its inverse (which is guaranteed to exist [35]), we get that

$$
\begin{aligned}
& \int_{0}^{1}\left(|Y|^{2}+|\omega|^{2}\right)\left(t, k_{x}, y, k_{z}\right) d y \\
\leq & C_{0} \mathrm{e}^{-2 \epsilon t} \int_{0}^{1}\left(|Y|^{2}+|\omega|^{2}\right)\left(0, k_{x}, y, k_{z}\right) d y
\end{aligned}
$$

Rafael Vazquez

DS-08-1170 
where $C_{0}>0$ is a constant depending on the kernels $K, \Gamma_{1}$ and $\Gamma_{2}$ and their inverses. Then writing $(u, W)$ in terms of $(Y, \omega)$ and bounding the norm of $V$ by the norm of $Y$ (using $Y=-V_{y}$ and Poincare's inequality), the result follows.

\subsection{Uncontrolled wave number analysis}

When $k_{x}^{2}+k_{z}^{2}>M$, the plant verifies the following equations

$$
\begin{aligned}
u_{t}= & \frac{-\alpha^{2} u+u_{y y}}{R e}-\beta(y) u-U_{y}^{e}(y) V-2 \pi k_{x} i p \\
& +2 \pi k_{z} i N \phi-N u, \\
V_{t}= & \frac{-\alpha^{2} V+V_{y y}}{R e}-\beta(y) V-p_{y}, \\
W_{t}= & \frac{-\alpha^{2} W+W_{y y}}{R e}-\phi W-2 \pi k_{z} i p-2 \pi k_{x} i N \phi-N W,
\end{aligned}
$$

the Poisson equation for the potential

$$
-\alpha^{2} \phi+\phi_{y y}=2 \pi i\left(k_{z} u-k_{x} W\right)
$$

the continuity equation

$$
2 \pi i k_{x} u+V_{y}+2 \pi k_{z} W=0
$$

and Dirichlet boundary conditions

$$
\begin{aligned}
& u\left(t, k_{x}, 0, k_{y}\right)=V\left(t, k_{x}, 0, k_{y}\right)=W\left(t, k_{x}, 0, k_{y}\right)=0, \\
& u\left(t, k_{x}, 1, k_{y}\right)=V\left(t, k_{x}, 1, k_{y}\right)=W\left(t, k_{x}, 1, k_{y}\right)=0, \\
& \phi\left(t, k_{x}, 0, k_{y}\right)=\phi\left(t, k_{x}, 1, k_{y}\right)=0 .
\end{aligned}
$$


Using (58), one gets the following equations for $Y$ and $\omega$.

$$
\begin{aligned}
Y_{t}= & \epsilon\left(-\alpha^{2} Y+Y_{y y}\right)-\beta(y) Y-2 \pi k_{x} i U_{y}^{e}(y) V \\
& +\alpha^{2} p-N Y \\
\omega_{t}= & \epsilon\left(-\alpha^{2} \omega+\omega_{y y}\right)-\beta(y) \omega-2 \pi k_{z} i U_{y}^{e}(y) V \\
& -\alpha^{2} N \phi-N \omega .
\end{aligned}
$$

The Poisson equation for the potential is, in terms of $\omega$,

$$
-\alpha^{2} \phi+\phi_{y y}=\omega
$$

Consider the Lyapunov function

$$
\Lambda=\int_{0}^{1} \frac{|u|^{2}+|V|^{2}+|W|^{2}}{2} d y
$$

where we write $\int_{0}^{1} f=\int_{0}^{1} f\left(k_{x}, y, k_{z}\right) d y$. The function $\Lambda$ is the $L^{2}$ norm (kinetic energy) of the perturbation velocity field (which is closely related to the turbulent kinetic energy).

Denote by $f^{*}$ the complex conjugate of $f$. Substituting $Y$ and $\omega$ from (59) into (105), we get

$$
\begin{aligned}
\Lambda= & \int_{0}^{1} 4 \pi^{2}\left[\frac{k_{x}^{2}|Y|^{2}+k_{z}^{2}|\omega|^{2}+k_{x} k_{z}\left(Y^{*} \omega+Y \omega^{*}\right)+k_{z}^{2}|Y|^{2}}{2 \alpha^{4}}\right. \\
& \left.+\frac{k_{x}^{2}|\omega|^{2}-k_{x} k_{z}\left(Y^{*} \omega+Y \omega^{*}\right)}{2 \alpha^{4}}\right]+\int_{0}^{1} \frac{|V|^{2}}{2} \\
= & \int_{0}^{1} \frac{|Y|^{2}+|\omega|^{2}+\alpha^{2}|V|^{2}}{2 \alpha^{2}} .
\end{aligned}
$$

Define then a new Lyapunov function,

$$
\Lambda_{1}=\alpha^{2} \Lambda=\int_{0}^{1} \frac{|Y|^{2}+|\omega|^{2}+\alpha^{2}|V|^{2}}{2} .
$$


The time derivative of $\Lambda_{1}$ can be estimated as follows,

$$
\begin{aligned}
\dot{\Lambda}_{1}= & -2 \epsilon \alpha^{2} \Lambda_{1}-\epsilon \int_{0}^{1}\left(\left|Y_{y}\right|^{2}+\left|\omega_{y}\right|^{2}+\alpha^{2}\left|V_{y}\right|^{2}\right) \\
& -N \int_{0}^{1}\left(|Y|^{2}+|\omega|^{2}\right)-\alpha^{2} N \int_{0}^{1} \frac{\phi^{*} \omega+\phi \omega^{*}}{2} \\
& +\int_{0}^{1} \pi i U_{y}^{e}(y)\left[V^{*}\left(2 k_{x} Y+k_{z} \omega\right)-V\left(2 k_{x} Y^{*}+k_{z} \omega^{*}\right)\right] \\
& +\alpha^{2} \int_{0}^{1} \frac{P^{*} Y+P Y^{*}-P_{y}^{*} V-P_{y} V^{*}}{2}
\end{aligned}
$$

For bounding (108), we use the following two lemmas.

Lemma 5.1. $-\alpha^{2} \int_{0}^{1} \frac{\phi^{*} \omega+\phi \omega^{*}}{2} \leq \int_{0}^{1}|\omega|^{2}$.

Proof. The term we want to estimate is

$$
-\alpha^{2} \int_{0}^{1} \frac{\phi^{*} \omega+\phi \omega^{*}}{2}
$$

Substituting $\alpha^{2} \phi$ from (104), (109) can be written as

$$
-\int_{0}^{1} \frac{\phi_{y y}^{*} \omega+\phi_{y y} \omega^{*}}{2}+\int_{0}^{1}|\omega|^{2} .
$$

Therefore, we need to prove that

$$
\int_{0}^{1}\left(\phi_{y y}^{*} \omega+\phi_{y y} \omega^{*}\right) \geq 0 .
$$

Substituting $\omega$ from equation (104) into (111), we get

$$
\begin{aligned}
& \int_{0}^{1}\left(\phi_{y y}^{*} \omega+\phi_{y y} \omega^{*}\right) \\
= & \int_{0}^{1}\left|\phi_{y y}\right|^{2}-\alpha^{2} \int_{0}^{1}\left(\phi_{y y}^{*} \phi+\phi_{y y} \phi^{*}\right) \\
= & \int_{0}^{1}\left|\phi_{y y}\right|^{2}+\alpha^{2} \int_{0}^{1}\left|\phi_{y}\right|^{2}
\end{aligned}
$$

which is nonnegative.

Rafael Vazquez 
Lemma 5.2. $\left|U_{y}^{e}(y)\right| \leq 4+H$.

Proof. Computing $U_{y}^{e}(y)$ from (15),

$$
U_{y}^{e}(y)=H \frac{\cosh (H y)-\cosh (H(1-y))}{2 \sinh H / 2-\sinh H}
$$

Calling $g_{1}(y)=\cosh (H y)-\cosh (H(1-y))$, since $g_{1}^{\prime}(y)=H(\sinh (H y)+\sinh (H(1-y)))$ is always positive for $y \in(0,1)$, the maximum must be in the boundaries. Therefore

$$
\left|U_{y}^{e}(y)\right| \leq g_{2}(H)=H \frac{\cosh H-1}{\sinh H-2 \sinh H / 2}
$$

One can rewrite $g_{2}$ as

$$
g_{2}=H \frac{\sinh H / 2}{\cosh H / 2-1}
$$

Since $g_{2}(0)=4$, it suffices to verify that $g_{2}^{\prime}(H) \leq 1$.

$$
g_{2}^{\prime}(H)=\frac{g_{3}}{g_{4}}=\frac{\sinh H / 2-H^{2} / 2}{\cosh H / 2-1} .
$$

This is equivalent to verify that $g_{3} \leq g_{4}$. Since $g_{3}(0)=g_{4}(0)=0$, it is enough that $g_{3}^{\prime} \leq g_{4}^{\prime}$, which follows from

$$
g_{3}^{\prime}=H / 2(\cosh H / 2-2 H) \leq H / 2(\sinh H / 2)=g_{4}^{\prime},
$$

because $\cosh x-4 x \leq \sinh x$.

Integrating by parts and applying Lemma 5.1,

$$
\begin{aligned}
\dot{\Lambda}_{1} \leq & -2 \epsilon \alpha^{2} \Lambda_{1}-\epsilon \int_{0}^{1}\left(\left|Y_{y}\right|^{2}+\left|\omega_{y}\right|^{2}+\alpha^{2}\left|V_{y}\right|^{2}\right) \\
& +\int_{0}^{1} \pi i U_{y}^{e}(y) V^{*}\left(k_{x} Y+k_{z} \omega\right) \\
& -\int_{0}^{1} \pi i U_{y}^{e}(y) V\left(k_{x} Y^{*}+k_{z} \omega^{*}\right)-N \int_{0}^{1}|Y|^{2} .
\end{aligned}
$$

Rafael Vazquez

DS-08-1170 
Using Lemma 5.2 to bound $U_{y}^{e}$ in (118),

$$
\begin{aligned}
\dot{\Lambda}_{1} \leq & -2 \epsilon\left(1+\alpha^{2}\right) \Lambda_{1}-N \int_{0}^{1}|Y|^{2} d y \\
& +2 \pi(4+H) \int_{0}^{1}\left(|V|\left(\left|k_{x}\right||Y|+\left|k_{z}\right||\omega|\right) d y\right. \\
\leq & \left(4+H-2 \epsilon\left(1+\alpha^{2}\right)\right) \Lambda_{1}
\end{aligned}
$$

where we have applied Young's and Poincare's inequalities. Hence, if $\alpha^{2} \geq \frac{4+H}{2 \epsilon}$,

$$
\dot{\Lambda}_{1} \leq-2 \epsilon \Lambda_{1}
$$

Dividing (120) by $\alpha^{2}$ and using (107), we get that

$$
\dot{\Lambda} \leq-2 \epsilon \Lambda
$$

and stability in the uncontrolled wave number range follows when $k_{x}^{2}+k_{z}^{2} \geq M^{2}$ for $M$ (conservatively) chosen as

$$
M \geq \frac{1}{2 \pi} \sqrt{\frac{(H+4) R e}{2}} .
$$

We summarize the result in the following proposition.

Proposition 5.2. For $k_{x}^{2}+k_{z}^{2} \geq M^{2}$ where $M \geq \frac{1}{2 \pi} \sqrt{\frac{(H+4) R e}{2}}$, the equilibrium $u \equiv V \equiv$ $W \equiv 0$ of the uncontrolled system (94)-(101) is exponentially stable in the $L^{2}$ sense, i.e.,

$$
\begin{aligned}
& \int_{0}^{1}\left(|u|^{2}+|V|^{2}+|W|^{2}\right)\left(t, k_{x}, y, k_{z}\right) d y \\
\leq & \mathrm{e}^{-2 \epsilon t} \int_{0}^{1}\left(\left|u_{0}\right|^{2}+\left|V_{0}\right|^{2}+\left|W_{0}\right|^{2}\right)\left(k_{x}, y, k_{z}\right) d y .
\end{aligned}
$$




\subsection{Main result}

Substituting (50), (53) and (87)-(88) in (44), and using the Fourier convolution theorem, we get the control laws in physical space, which can be expressed compactly as

$$
\begin{aligned}
\left(\begin{array}{c}
U_{c} \\
W_{c} \\
\Phi_{c}
\end{array}\right)= & \int_{-\infty}^{\infty} \int_{0}^{1} \int_{-\infty}^{\infty} \Sigma(x-\xi, \eta, z-\zeta) \\
& \times\left(\begin{array}{c}
u(\xi, \eta, \zeta) \\
W(\xi, \eta, \zeta)
\end{array}\right) d \xi d \eta d \zeta
\end{aligned}
$$

where

$$
\begin{aligned}
\Sigma(\xi, \eta, \zeta)= & \int_{-\infty}^{\infty} \int_{-\infty}^{\infty} \Sigma\left(k_{x}, \eta, k_{z}\right) \\
& \times \chi\left(k_{x}, k_{z}\right) \mathrm{e}^{2 \pi i\left(k_{x} \xi+k_{z} \zeta\right)} d k_{z} d k_{x},
\end{aligned}
$$

and

$$
\Sigma=\left(\begin{array}{cc}
K^{U u}\left(k_{x}, 1, \eta, k_{z}\right) & K^{U W}\left(k_{x}, 1, \eta, k_{z}\right) \\
K^{W u}\left(k_{x}, 1, \eta, k_{z}\right) & K^{W W}\left(k_{x}, 1, \eta, k_{z}\right) \\
\frac{2 \pi i k_{z}}{\alpha} \sinh (\alpha(1-\eta)) & -\frac{2 \pi i k_{x}}{\alpha} \sinh (\alpha(1-\eta))
\end{array}\right) .
$$

Control law $V_{c}$ is a dynamic feedback law computed as the solution of the following forced parabolic equation

$$
\left(V_{c}\right)_{t}=\frac{\left(V_{c}\right)_{x x}+\left(V_{c}\right)_{z z}}{R e}-N V_{c}+g(t, x, z)
$$

where $g(t, x, z)$ is defined as

$$
\begin{aligned}
g= & \int_{-\infty}^{\infty} \int_{-\infty}^{\infty}\left[\int_{0}^{1} g_{V}(x-\xi, \eta, z-\zeta) V(\xi, \eta, \zeta) d \eta\right. \\
& +g_{W}(x-\xi, z-\zeta)\left(W_{y}(\xi, 0, \zeta)-W_{y}(\xi, 1, \zeta)\right) \\
& \left.+g_{u}(x-\xi, z-\zeta)\left(u_{y}(\xi, 0, \zeta)-u_{y}(\xi, 1, \zeta)\right)\right] d \xi d \zeta
\end{aligned}
$$


and

$$
\begin{aligned}
g_{u}= & \int_{-\infty}^{\infty} \int_{-\infty}^{\infty} 2 \pi i \frac{k_{x}}{R e} \chi\left(k_{x}, k_{z}\right) \mathrm{e}^{2 \pi i\left(k_{x} \xi+k_{z} \zeta\right)} d k_{z} d k_{x} \\
g_{V}= & \int_{-\infty}^{\infty} \int_{-\infty}^{\infty} \cosh (\alpha(1-\eta))\left(N+4 \pi k_{x} i U_{y}^{e}(\eta)\right) \\
& \times \chi\left(k_{x}, k_{z}\right) \mathrm{e}^{2 \pi i\left(k_{x} \xi+k_{z} \zeta\right)} d k_{z} d k_{x}, \\
g_{W}= & \int_{-\infty}^{\infty} \int_{-\infty}^{\infty} 2 \pi i \frac{k_{z}}{R e} \chi\left(k_{x}, k_{z}\right) \mathrm{e}^{2 \pi i\left(k_{x} \xi+k_{z} \zeta\right)} d k_{z} d k_{x} .
\end{aligned}
$$

As in $[5,6]$, considering all wave numbers and using Proposition 5.1 and Proposition 5.2, the following result holds regarding the convergence of the closed-loop system.

Theorem 1. Consider the system (21)-(30) with control laws (124)-(131). Then the equilibrium profile $u \equiv V \equiv W \equiv 0$ is asymptotically stable in the $L^{2}$ norm, i.e.,

$$
\begin{aligned}
& \int_{-\infty}^{\infty} \int_{0}^{1} \int_{-\infty}^{\infty}\left(u^{2}+V^{2}+W^{2}\right)(t) d x d y d z \\
\leq & C_{2} \mathrm{e}^{-2 \epsilon t} \int_{-\infty}^{\infty} \int_{0}^{1} \int_{-\infty}^{\infty}\left(u_{0}^{2}+V_{0}^{2}+W_{0}^{2}\right)(x, y, z) d x d y d z .
\end{aligned}
$$

where $C_{2}=\max \left\{C_{1}, 1\right\} \geq 0$.

We have assumed in the above result that the closed loop linearized system is well-posed and that the velocity and electromagnetic field equations have at least $L^{2}$ solutions. See [40] for some mathematical considerations on the well-posedness of MHD problems.

Remark 5.2. In case that $N=0$, meaning that either there is no imposed magnetic field or the fluid is nonconducting, Equations (2)-(4) are the Navier-Stokes equations. Some physical insight can be gained analyzing this case. In the context of hydrodynamic stability theory, the linearized observer error system written in $(Y, \omega)$ variables verify equations analogous to the classical Orr-Sommerfeld-Squire equations. These are Equations (64)-(65) for controlled Rafael Vazquez 
wave numbers and Equations (102)-(103) for uncontrolled wave numbers. As in [6], we use the backstepping transformations (76)-(77) not only to stabilize (damping the system using gain $l$ ) but also to decouple the system (using gains $\Gamma_{1}, \Gamma_{2}$ ) in the small wave number range, where non-normality effects are more severe. Even if the linearized system is stable, nonnormality produces large transient growths [3, 37], which enhanced by nonlinear effects may allow the system to go far away from the origin, activating the mechanism of transition to turbulence. This warrants the use of extra gains to map the system into two uncoupled heat equations (72)-(73).

Remark 5.3. As in [5, Theorem 2], some properties of the feedback control laws can be derived. The most important properties of the feedback laws from the point of view of implementation are the spatial decay of the kernels and conservation of mass. Spatial decay means that the function $\Sigma(x, y, z)$ appearing in (124) rapidly decrease as $x$ or $z$ grow, and that implies that the control law mostly needs information about the states close to the actuation point. This suggest that our control laws could be approximately implemented by an array of discrete actuators, each of them only requiring information only from the flow in its vicinity. Conservation of mass is derived by studying the behavior of the control laws at wave numbers $k_{x}=k_{z}=0$, and can be mathematically expressed as

$$
\int_{-\infty}^{\infty} \int_{-\infty}^{\infty} V_{c}(x, z) d x d z=0
$$

Physically, it means that the actuators do not need to add or substract mass from the flow to stabilize it, which is a very desirable property for a possible implementation. 


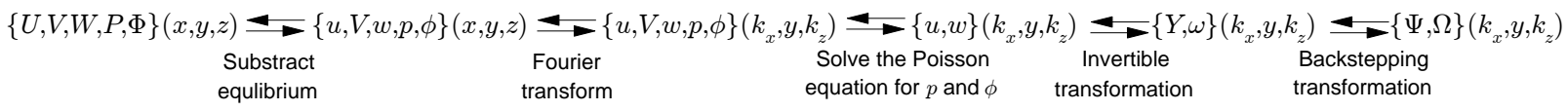

$$
\begin{aligned}
& \begin{array}{c}
\text { Apply } V_{c} \text { and } \Phi_{c} \\
\text { controllers }
\end{array}
\end{aligned}
$$

Figure 3: The chain of transformations used to design the control laws. Note that all transformations are invertible.

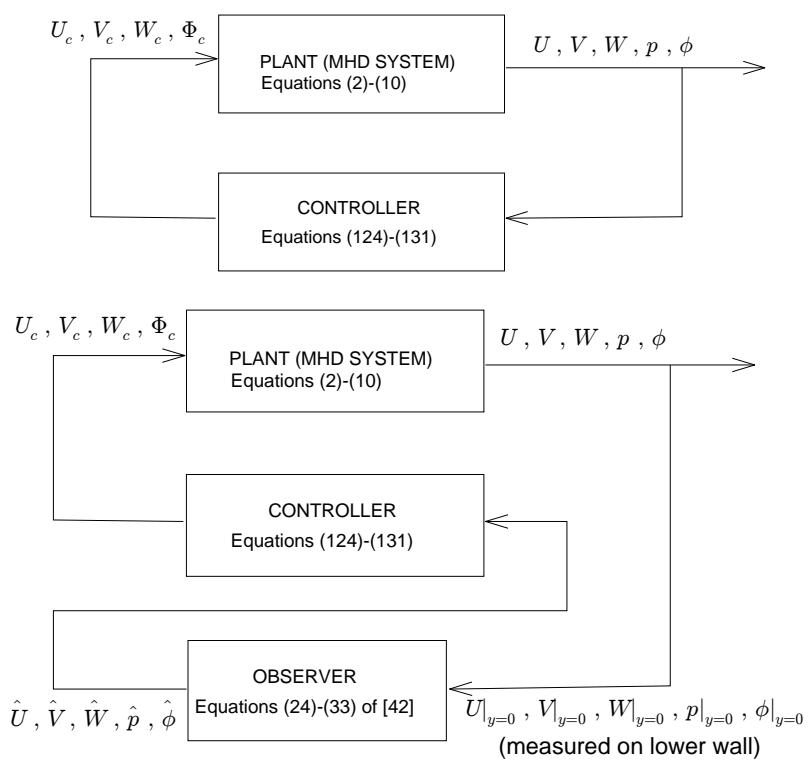

Figure 4: A block diagram showing the structure of the controller. (Top) Full-state controller. (Bottom) Output-feedback controller (with measurements on the lower wall).

\section{Concluding Remarks}

In this paper we have stabilized the Hartmann flow, a benchmark MHD model with several potential applications. We have used the backstepping method, that allows to compute the control kernels without needing to discretize the system. Our solution is summarized in control laws (124)-(131) and Theorem 1. The feedback kernels given by (89)-(90) and calculated from (78)-(84) can be computed beforehand.

The design of the control laws (124)-(131) was based on using several invertible transfor- 
mations to simplify the problem and then go back to obtain the solution in physical space. Fig. 3 summarizes all the transformations that were used. The structure of the controller is shown in Fig. 4 (top).

These feedbacks laws require full-state knowledge. In [32] we presented an observer for estimation of velocity and electromagnetic fields of the Hartmann flow, based on boundary measurement of pressure, current and skin friction. Such an observer can be used together with the control laws (124)-(131) to obtain an output feedback stabilizing boundary controller that only needs boundary measurements; a block diagram showing the structure of the proposed output feedback controller is shown in Fig. 4 (bottom).

This work can be used as the starting point to also solve the problems of motion planning and trajectory tracking, which are of interest in engineering applications. The problem has been solved in the case of non-conducting fluids [41] using the backstepping method.

Our result uses the linear backstepping control method for parabolic PDEs and thus requires linearization of the MHD equations as a first step. Hence, Theorem 1 only holds for initial conditions close enough to the equilibrium profile. If one considers instead the fully nonlinear MHD equations, the problem is extremely challenging not only because of the nonlinearity itself, but also because the plant becomes coupled in the wave number space. The nonlinearity is of bilinear type and recent developments that extend the backstepping method to nonlinear parabolic PDEs using Volterra series [42, 43] and to the viscouse Burgers equation $[44,45]$ are potentially applicable, however the method has to be extended to consider the coupling between different wave numbers. 


\section{References}

[1] Hartmann, J., 1937, "Theory of the laminar flow of an electrically conductive liquid in a homogeneous magnetic field," Det Kgl. Danske Vidensk-abernes Selskab Mathematiskfysiske Meddelelser XV, 6, pp. 1-27.

[2] Muller, U. and Buhler, L., 2001, Magnetofluiddynamics in Channels and Containers, Springer, Berlin.

[3] Schmid, P. J. and Henningson, D. S., 2001, Stability and Transition in Shear Flows, Springer, New York.

[4] Hogberg, M., Bewley, T. R., and Henningson, D. S., 2003, "Linear feedback control and estimation of transition in plane channel flow," Journal of Fluid Mechanics, 481, pp. $149-175$.

[5] Vazquez, R. and Krstic, M., 2007, "A closed-form feedback controller for stabilization of the linearized 2D Navier-Stokes Poiseuille flow," IEEE Transactions on Automatic Control, 52, pp. 2298-2312.

[6] Cochran, J., Vázquez, R., and Krstic, M., 2006, "Backstepping boundary control of Navier-Stokes channel flow: a 3D extension," Proceedings of the 2006 American Control Conference.

[7] Triggiani, R., 2007, "Stability enhancement of a 2-D linear Navier-Stokes channel flow by a 2-D, wall-normal boundary controller," DCDS-B, 8(2), pp. 279-314. 
[8] Aamo, O. M. and Krstic, M., 2002, Flow Control by Feedback: Stabilization and Mixing, Springer, New York.

[9] Balogh, A., Liu, W.-J., and Krstic, M., 2001, "Stability enhancement by boundary control in 2D channel flow," IEEE Transactions on Automatic Control, 46, pp. 16961711.

[10] Baker, J., Armaou, A., and Christofides, P., 2000, "Nonlinear control of incompressible fluid flow: application to Burgers' equation and 2D channel flow," J. Math. Anal. Appl., 252, pp. 230-255.

[11] Vladimirov, V. and Ilin, K., 1998, "The three-dimensional stability of steady MHD flows of an ideal fluid," Physics of Plasmas, 5(12), pp. 4199-4204.

[12] Takashima, M., 1996, "The stability of the modified plane Poisseuille flow in the presence of a transverse magnetic field," Fluid Dynamics Research, 17, pp. 293-310.

[13] Krasnov, D., Zienicke, E., Zikanov, O., Boeck, T., and Thess, A., 2004, "Numerical study of the instability of the Hartmann layer," Journal of Fluid Mechanics, 504, pp. $183-211$.

[14] Lock, R., 1955, "The stability of the flow of an electrically conducting fluid between parallel planes under a transverse magnetic field," Proceedings of Royal Society of London A, 233, p. 105.

[15] Albrecht, T., Metzkes, H., Grundmann, R., Mutschke, G., and Gerbeth, G., 2008, "Tollmien-Schlichting wave damping by a streamwise oscillating Lorentz force," Magnetohydrodynamics, 44(3), pp. 205-222. 
[16] Pang, J. and Choi, K.-S., 2004, "Turbulent drag reduction by Lorentz force oscillation," Phys. Fluids, 16(5), p. L35.

[17] Breuer, K. and Park, J., 2004, "Actuation and control of a turbulent channel flow using lorentz forces," Phys. Fluids, 16(4), p. 897.

[18] Spong, E., Reizes, J., and Leonardi, E., 2005, "Efficiency improvements of electromagnetic flow control," Heat and Fluid Flow, 26, pp. 635-655.

[19] Berger, W., Kim, J., Lee, C., and Lim, J., 2000, "Turbulent boundary layer control utilizing the lorentz force," Phys. Fluids, 12, p. 631.

[20] H. Choi, P. M. and Kim, J., 1994, "Active turbulence control for drag reduction in wallbounded flows," Journal of Fluid Mechanics, 262, p. 75.

[21] Baker, J. and Christofides, P., 2002, "Drag reduction in transitional linearized channel flow using distributed control," Int. J. Contr., 75, pp. 1213-1218.

[22] Airau, C. and Castets, M., 2004, "On the amplification of small disturbances in a channel flow with a normal magnetic field," Physics of Fluids, 16, pp. 2991-3005.

[23] Debbagh, K., Cathalifaud, P., and Airiau, C., 2007, "Optimal and robust control of small disturbances in a channel flow with a normal magnetic field," Physics of Fluids, 19(1), pp. 014103.1-014103.14.

[24] Thibault, J.-P. and Rossi, L., 2003, "Electromagnetic flow control: characteristic numbers and flow regimes of a wall-normal actuator," J. Phys. D: Appl. Phys., 36, pp. $2559-2568$. 
[25] Singh, S. and Bandyopadhyay, P., 1997, "Linear feedback control of boundary layer using electromagnetic microtiles," Transactions of ASME, 119, pp. 858-858.

[26] Barbu, V., Popa, C., Havarneanu, T., and Sritharan, S., 2003, "Exact controllability of magneto-hydrodynamic equations," Communications on Pure and Applied Mathematics, 56(6), pp. 732-783.

[27] Sritharan, S., Barbu, V., Havarneanu, T., and Popa, C., 2005, "Advances in differential equations," IEEE Transactions on Automatic Control, 10(5), pp. 481-504.

[28] Dietiker, J.-F. and Hoffmann, K., 2002, "Backstepping boundary control of NavierStokes channel flow: a 3D extension," AIAA 2002-0130, 40th Aerospace Sciences Meeting and Exhibit.

[29] Schuster, E. and Krstic, M., 2003, "Inverse optimal boundary control for mixing in magnetohydrodynamic channel flows," Proceedings of the 2003 CDC.

[30] Schuster, E., Luo, L., and Krstic, M., 2008, "MHD channel flow control in 2D: Mixing enhancement by boundary feedback," Automatica, 44, pp. 2498-2507.

[31] Bandyopadhyay, P. R. and Castano, J. M., 1996, "Micro-tiles for electromagnetic turbulence control in saltwater — preliminary investigations," ASME Fluids Engineering Division Conference, vol. 2, p. 53.

[32] Vazquez, R., Schuster, E., and Krstic, M., 2008, "Magnetohydrodynamic state estimation with boundary sensors," Automatica, 44, pp. 2517-2527. 
[33] Xu, C., Schuster, E., Vazquez, R., and Krstic, M., 2008, "Stabilization of linearized 2D magnetohydrodynamic channel flow by backstepping boundary control," Systems and Control Letters, 57, pp. 805-812.

[34] Bamieh, B., Paganini, F., and Dahleh, M. A., 2000, "Distributed control of spatiallyinvariant systems," IEEE Transactions on Automatic Control, 45, pp. 1091-1107.

[35] Smyshlyaev, A. and Krstic, M., 2004, "Closed form boundary state feedbacks for a class of partial integro-differential equations," IEEE Transactions on Automatic Control, 49, pp. 2185-2002.

[36] Jovanovic, M. and Bamieh, B., 2005, "Componentwise energy amplification in channel flows," Journal of Fluid Mechanics, 543, pp. 145-183.

[37] Reddy, S. C., Schmid, P. J., and Henningson, D. S., 1993, "Pseudospectra of the OrrSommerfeld operator," SIAM J. Appl. Math., 53(1), pp. 15-47.

[38] Lee, D. and Choi, H., 2001, "Magnetohydrodynamic turbulent flow in a channel at low magnetic Reynolds number," Journal of Fluid Mechanics, 439, pp. 367-394.

[39] Vazquez, R., Trelat, E., and Coron, J.-M., 2008, "Control for fast and stable laminar-tohigh-Reynolds-numbers transfer in a 2D Navier-Stokes channel flow," DCDS-B, 10, pp. 925-956.

[40] Sermange, M. and Temam, R., 1983, "Some mathematical questions related to the MHD equations," Communications on Pure and Applied Mathematics, 36, pp. 635-664. 
[41] Cochran, J. and Krstic, M., 2008, "Motion planning and trajectory tracking for the 3-D Poiseuille flow," Journal of Fluid Mechanics, to appear.

[42] Vazquez, R. and Krstic, M., 2008, "Control of 1-D parabolic PDEs with Volterra nonlinearities - Part i: Design," Automatica, 44, pp. 2778-2790.

[43] Vazquez, R. and Krstic, M., 2008, "Control of 1-D parabolic PDEs with Volterra nonlinearities - Part ii: Analysis," Automatica, 44, pp. 2791-2803.

[44] Krstic, M., Magnis, L., and Vazquez, R., 2008, "Nonlinear stabilization of shock-like unstable equilibria in the viscous Burgers PDE," IEEE Transactions on Automatic Control, 53, pp. 1678-1683.

[45] Krstic, M., Magnis, L., and Vazquez, R., 2008, "Nonlinear control of the viscous Burgers equation: Trajectory generation, tracking, and observer design," Journal of Dynamic Systems, Measurement, and Control, to appear. 


\section{List of Figure Captions}

1. Figure 1: Hartmann Flow.

2. Figure 2: Streamwise equilibrium velocity $U^{e}(y)$ (left) and $U_{y}^{e}(y)$ (right), for different values of $H$. Solid, $H=0$; dash-dotted, $H=10$; dashed, $H=50$.

3. Figure 3: The chain of transformations used to design the control laws. Note that all transformations are invertible.

4. Figure 4: A block diagram showing the structure of the controller. (Top) Full-state controller. (Bottom) Output-feedback controller (with measurements on the lower wall). 